DOI: 10.2478/awutm-2013-0018

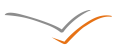

VERSITA
Analele Universităţii de Vest,

Timişoara

Seria Matematică - Informatică

LI, 2, (2013), 89- 113

\title{
On the Bounds for the Derivatives of the Solutions of the Linear Volterra Integral Equations
}

\author{
İsmet Özdemir and Ö. Faruk Temizer
}

Abstract. Some bounds about the solution of the linear Volterra integral equations of the second type of the form

$$
f(t)=1-\int_{0}^{t} K(t-\tau) f(\tau) d \tau=1-K * f
$$

with unit source term and positive monotonically increasing convolution kernel were obtained in $[5,6,8-10]$. The boundedness of functions $f^{\prime}, f^{\prime \prime}, \ldots, f^{(n)},(n \in \mathbb{N})$ defined on the infinite interval $[0, \infty)$ were shown in $[10,12]$. In this paper, it is shown that the results in $[10,12]$ for an equation in the same form can also be derived under different conditions than those of $[10,12]$.

AMS Subject Classification (2000). Primary: 45D05, Secondary: 45E10.

Keywords. Linear Volterra integral equations with convolution kernel, Equivalence theorem, Convolution theorem.

\section{Introduction}

Volterra integral equations arise in great many branches of science. For example they are models of epidemic diffusion, population dynamics, reaction- 
diffusion in small cells and in general of evolutionary phenomena incorporating memory, [1].

In the study of linear viscoelastic materials, from measurements of the relaxation modulus $G(t)$, approximations to the corresponding creep compliance (retardation) modulus $J(t)$ are determined by solving the convolution interconversion equation

$$
\int_{0}^{t} G(t-\tau) J(\tau) d \tau=t, \quad t \geq 0
$$

where $G \in C^{1}[0, \infty), G(t)>0$ and $G^{\prime}(t)<0$ for all $t$ such that $0 \leq t<\infty$, [2]. Differentiation of (1.1) leads to

$$
J(t)=\frac{1}{G(0)}-\frac{1}{G(0)} \int_{0}^{t} G^{\prime}(t-\tau) J(\tau) d \tau
$$

(1.2) is the linear second type convolution Volterra integral equation.

An integral equation of the form

$$
f(t)=\phi(t)-\int_{0}^{t} K(t-\tau) f(\tau) d \tau=\phi(t)-K * f
$$

is known as the second type linear Volterra integral equation with convolution kernel, where $\phi$ is the source term and $K$ is kernel which are the known functions, $f$ is an unknown function, [13, p.23]. Such equations are important in many applications, including rheology, risk theory and various applications of renewal theory including branching processes, queuing theory and inventory analysis, [4]. Some properties of the solution of the integral equation (1.3) are investigated on the infinite interval, [7,11].

The way of obtaining a new equation which is equivalent to the equation of the form (1.3) is given by Theorem $A$ named as Equivalence Theorem as follows:

Theorem 1.1.(Equivalence Theorem). [5, Theorem 1.1.1] If

(1) $K \in C^{1}[0, \infty)$,

(2) $\phi$ is locally integrable,

then the equation (1.3) is equivalent to

$$
f(t)=\psi(t)-\int_{0}^{t} L(t-\tau) f(\tau) d \tau,
$$


where

$$
\begin{gathered}
\psi(t)=\phi(t)+\int_{0}^{t} g^{\prime}(t-\tau) \phi(\tau) d \tau, \\
L(t)=g^{\prime}(t)+a g(t)+\int_{0}^{t} g(t-\tau) K^{\prime}(\tau) d \tau,
\end{gathered}
$$

where $a=K(0), g$ is any function such that $g \in C^{1}[0, \infty)$ and $g(0)=1$.

The sufficient conditions which enable to derive the solution of (1.3) in terms of the solution of the equation

$$
h(t)=1-\int_{0}^{t} K(t-\tau) h(\tau) d \tau=1-K * h
$$

are given by the Convolution Theorem.

Theorem 1.2.(Convolution Theorem). [3, pp. 229-230] Let the conditions

(1) $\phi^{\prime}(t)$ exists for $0 \leq t \leq T, \int_{0}^{T}\left|\phi^{\prime}(t)\right| d t<\infty, \quad(T>0)$,

(2) $\int_{0}^{T}|K(t)| d t<\infty$

hold, then the solution of equation (1.3) is given of the form

$$
\begin{aligned}
f(t) & =h(t) \phi(0)+\int_{0}^{t} h(t-\tau) \phi^{\prime}(\tau) d \tau \\
& =h(t) \phi(0)+h * \phi^{\prime}, \quad(0 \leq t \leq T),
\end{aligned}
$$

where $h(t)$ is the solution of (1.4).

Therefore, if $h$ is known, so is $f$. Or if the properties of $h$ are known, we may be able to obtain certain properties of $f$ by (1.5).

We assume throughout that $t \in[0, \infty)$ and $n \in \mathbb{N}_{2}=\{2,3, \ldots\}$.

\section{The Main Results}

Theorem C. Let us consider the equation

$$
f(t)=1-\int_{0}^{t} K(t-\tau) f(\tau) d \tau=1-K * f
$$

If the conditions 
(1) $K \in C^{2}[0, \infty), K(t)>0, K^{\prime}(t)>0$ and $K^{\prime \prime}(t) \leq 0$ for all $t$,

(2) $4 b \leq a^{2}$

hold, then the solution of (2.1) satisfies the inequalities $\left|f^{\prime}(t)\right| \leq 3 a / 2$ and $\left|f^{\prime \prime}(t)\right| \leq\left(3 a^{2}+4 b\right) / 2$ for all $t$, where $a=K(0)$ and $b=K^{\prime}(0)$.

Proof. Firstly, we can assert that $f$ provides the inequality $|f(t)| \leq 1$ for all $t$, [6, Theorem B]. By choosing $g(t)=e^{-\gamma t}\left(\gamma=\gamma_{0}=a / 2\right)$ in Theorem $A$ (Equivalence Theorem), we get the equivalent equation of (2.1) of the form

$$
f(t)=e^{-\gamma t}-\int_{0}^{t} L(t-\tau) f(\tau) d \tau=e^{-\gamma t}-L * f
$$

where

$$
L(t)=(a-\gamma) e^{-\gamma t}+K^{\prime} * e^{-\gamma t}=\left(\frac{a}{2}\right) e^{-\gamma t}+K^{\prime} * e^{-\gamma t}>0
$$

and thus,

$$
\begin{aligned}
L^{\prime}(t) & =\left(\gamma^{2}-a \gamma+b\right) e^{-\gamma t}+K^{\prime \prime} * e^{-\gamma t} \\
& =\left(-\frac{a^{2}}{4}+b\right) e^{-\gamma t}+K^{\prime \prime} * e^{-\gamma t} \leq 0
\end{aligned}
$$

for all $t$. On the other hand, differentiation of the equivalent equation (2.2) leads to

$$
f^{\prime}(t)=-\gamma e^{-\gamma t}-L(0) f(t)-L^{\prime} * f .
$$

Taking $\gamma=\gamma_{0}=a / 2$, the inequality

$$
\begin{aligned}
\left|f^{\prime}(t)\right| & \leq \gamma+\frac{a}{2}|f(t)|+\int_{0}^{t}\left|L^{\prime}(t-\tau)\right||f(\tau)| d \tau \\
& \leq \gamma+\frac{a}{2}-\int_{0}^{t} L^{\prime}(t-\tau) d \tau=\gamma+\frac{a}{2}+L(0)-L(t) \\
& =\frac{a}{2}+\frac{a}{2}+\frac{a}{2}-L(t)=\frac{3 a}{2}-L(t) \leq \frac{3 a}{2}
\end{aligned}
$$

is obtained for all $t$. From (2.1),

$$
f^{\prime}(t)=-a f(t)-K^{\prime} * f
$$

and so

$$
f^{\prime \prime}(t)=-a f^{\prime}(t)-b f(t)-K^{\prime \prime} * f .
$$


By the inequalities $|f(t)| \leq 1$ and (2.3), we have

$$
\begin{aligned}
\left|f^{\prime \prime}(t)\right| & \leq a\left|f^{\prime}(t)\right|+b|f(t)|+\int_{0}^{t}\left|K^{\prime \prime}(t-\tau)\right||f(\tau)| d \tau \\
& \leq a\left(\frac{3 a}{2}\right)+b-\int_{0}^{t} K^{\prime \prime}(t-\tau) d \tau \\
& =\frac{3 a^{2}}{2}+b+K^{\prime}(0)-K^{\prime}(t) \\
& =\frac{3 a^{2}}{2}+2 b-K^{\prime}(t) \leq \frac{1}{2}\left(3 a^{2}+4 b\right)
\end{aligned}
$$

for all $t$.

Theorem 1. Let us consider the equation

$$
f_{1}(t)=1-\int_{0}^{t} K_{1}(t-\tau) f_{1}(\tau) d \tau=1-K_{1} * f_{1} .
$$

If

(1) $K_{1} \in C^{3}[0, \infty), K_{1}(t)>0, K_{1}^{\prime}(t)>0, K_{1}^{\prime \prime}(t)>0$ and $K_{1}^{\prime \prime \prime}(t) \leq 0$ for all $t$,

(2) $a_{10}^{2}<4 a_{11}$,

(3) $3 a_{11} \leq a_{10}^{2}$,

(4) $2 a_{10}^{3}-9 a_{10} a_{11}+27 a_{12} \leq 0$,

then the solution of (2.4) satisfies the inequalities

$$
\left|f_{1}^{\prime}(t)\right| \leq \frac{7 a_{10}}{3},\left|f_{1}^{\prime \prime}(t)\right| \leq \frac{1}{9}\left(7 a_{10}^{2}+36 a_{11}\right)
$$

and

$$
\left|f_{1}^{\prime \prime \prime}(t)\right| \leq \frac{1}{9}\left(7 a_{10}^{3}+57 a_{10} a_{11}+36 a_{12}\right)
$$

for all $t$, where $a_{10}=K_{1}(0), a_{11}=K_{1}^{\prime}(0)$ and $a_{12}=K_{1}^{\prime \prime}(0)$.

Proof. One can immediately see from Theorem 3.1 of [6] that $f_{1}$ provides the inequality $\left|f_{1}(t)\right| \leq 2$ for all $t$. By choosing $g(t)=e^{-\gamma t}$ and $\gamma=\gamma_{1}=a_{10} / 3$ in the Equivalence Theorem, we get the equivalent equation to (2.4) of the form

$$
f_{1}(t)=e^{-\gamma t}-\int_{0}^{t} L_{1}(t-\tau) f_{1}(\tau) d \tau=e^{-\gamma t}-L_{1} * f_{1},
$$


where

$$
L_{1}(t)=\left(a_{10}-\gamma\right) e^{-\gamma t}+K_{1}^{\prime} * e^{-\gamma t}
$$

Thus,

$$
L_{1}^{\prime}(t)=\left(\gamma^{2}-a_{10} \gamma+a_{11}\right) e^{-\gamma t}+K_{1}^{\prime \prime} * e^{-\gamma t}
$$

and

$$
L_{1}^{\prime \prime}(t)=\left(-\gamma^{3}+a_{10} \gamma^{2}-a_{11} \gamma+a_{12}\right) e^{-\gamma t}+K_{1}^{\prime \prime \prime} * e^{-\gamma t} .
$$

It is known that $L_{1}$ satisfies all of the conditions of Theorem $C$ from the proof of Theorem 3.1 of [6]. Hence, by Theorem $C$, the first two derivatives of the solution of the equation $h_{1}(t)=1-L_{1} * h_{1}$ verify the inequalities

$$
\left|h_{1}^{\prime}(t)\right| \leq \frac{3 L_{1}(0)}{2}=\frac{3\left(a_{10}-\gamma\right)}{2}=a_{10}=y_{11}
$$

and

$$
\begin{aligned}
\left|h_{1}^{\prime \prime}(t)\right| & \leq \frac{1}{2}\left[3\left(L_{1}(0)\right)^{2}+4 L_{1}^{\prime}(0)\right] \\
& =\frac{1}{2}\left[3\left(a_{10}-\gamma\right)^{2}+4\left(\gamma^{2}-a_{10} \gamma+a_{11}\right)\right] \\
& =\frac{1}{9}\left(2 a_{10}^{2}+18 a_{11}\right)=y_{12}
\end{aligned}
$$

for all $t$. By the Convolution Theorem, the solution $f_{1}$ of the equivalent equation (2.5) can be written by means of $h_{1}$ of the form

$$
f_{1}(t)=h_{1}(t)-\gamma h_{1} * e^{-\gamma t} .
$$

Thus, we have by (2.6) that

$$
f_{1}^{\prime}(t)=h_{1}^{\prime}(t)-\gamma\left(h_{1}(0) e^{-\gamma t}+h_{1}^{\prime} * e^{-\gamma t}\right)
$$

which yields

$$
f_{1}^{\prime \prime}(t)=h_{1}^{\prime \prime}(t)+\gamma^{2} e^{-\gamma t}-\gamma h_{1}^{\prime}(0) e^{-\gamma t}-\gamma h_{1}^{\prime \prime} * e^{-\gamma t} .
$$

By (2.7) and (2.8),

$$
\begin{aligned}
\left|f_{1}^{\prime}(t)\right| & \leq\left|h_{1}^{\prime}(t)\right|+\gamma+\gamma \int_{0}^{t}\left|h_{1}^{\prime}(t-\tau)\right| e^{-\gamma \tau} d \tau \\
& \leq y_{11}+\gamma+\gamma y_{11} \int_{0}^{t} e^{-\gamma \tau} d \tau=y_{11}+\gamma+y_{11}-y_{11} e^{-\gamma t} \\
& =2 y_{11}+\gamma-y_{11} e^{-\gamma t} \leq 2 y_{11}+\gamma=\frac{7 a_{10}}{3}=x_{11}
\end{aligned}
$$


and

$$
\begin{aligned}
\left|f_{1}^{\prime \prime}(t)\right| & \leq\left|h_{1}^{\prime \prime}(t)\right|+\gamma^{2}+\gamma\left|h_{1}^{\prime}(0)\right|+\gamma \int_{0}^{t}\left|h_{1}^{\prime \prime}(t-\tau)\right| e^{-\gamma \tau} d \tau \\
& \leq y_{12}+\gamma^{2}+\gamma y_{11}+\gamma y_{12} \int_{0}^{t} e^{-\gamma \tau} d \tau \\
& =2 y_{12}+\gamma^{2}+\gamma y_{11}-y_{12} e^{-\gamma t} \\
& \leq 2 y_{12}+\gamma^{2}+\gamma y_{11}=\frac{1}{9}\left(8 a_{10}^{2}+36 a_{11}\right)=b_{12}
\end{aligned}
$$

for all $t$ and $\gamma=\gamma_{1}=a_{10} / 3$.

On the other hand, differentiation of the equivalent equation (2.5) leads to

$$
f_{1}^{\prime \prime}(t)=\gamma^{2} e^{-\gamma t}-L_{1}(0) f_{1}^{\prime}(t)-L_{1}^{\prime}(0) f_{1}(t)-L_{1}^{\prime \prime} * f_{1} .
$$

Taking $\gamma=\gamma_{1}=a_{10} / 3$, the inequality

$$
\begin{aligned}
\left|f_{1}^{\prime \prime}(t)\right| \leq & \gamma^{2}+L_{1}(0)\left|f_{1}^{\prime}(t)\right|+L_{1}^{\prime}(0)\left|f_{1}(t)\right|+ \\
& +\int_{0}^{t}\left|L_{1}^{\prime \prime}(t-\tau)\right|\left|f_{1}(\tau)\right| d \tau \\
\leq & \gamma^{2}+\left(a_{10}-\gamma\right) x_{11}+\left(\gamma^{2}-a_{10} \gamma+a_{11}\right) 2-2 \int_{0}^{t} L_{1}^{\prime \prime}(\tau) d \tau \\
= & \gamma^{2}+\left(a_{10}-\gamma\right) x_{11}+4\left(\gamma^{2}-a_{10} \gamma+a_{11}\right)-2 L_{1}^{\prime}(t) \\
\leq & \gamma^{2}+\left(a_{10}-\gamma\right) x_{11}+4\left(\gamma^{2}-a_{10} \gamma+a_{11}\right) \\
= & \frac{1}{9}\left(7 a_{10}^{2}+36 a_{11}\right)=c_{12}
\end{aligned}
$$

is obtained for all $t$. From (2.10) and (2.11), we get the inequality

$$
\left|f_{1}^{\prime \prime}(t)\right| \leq \min \left\{b_{12}, c_{12}\right\}=c_{12}=\frac{1}{9}\left(7 a_{10}^{2}+36 a_{11}\right)=x_{12}
$$

for all $t$. From (2.4),

$$
f_{1}^{\prime \prime \prime}(t)=-a_{10} f_{1}^{\prime \prime}(t)-a_{11} f_{1}^{\prime}(t)-a_{12} f_{1}(t)-K_{1}^{\prime \prime \prime} * f_{1} .
$$

By the inequalities $\left|f_{1}(t)\right| \leq 2,(2.9)$ and (2.12), we have the inequality

$$
\begin{aligned}
\left|f_{1}^{\prime \prime \prime}(t)\right| & \leq a_{10}\left|f_{1}^{\prime \prime}(t)\right|+a_{11}\left|f_{1}^{\prime}(t)\right|+a_{12}\left|f_{1}(t)\right|+\int_{0}^{t}\left|K_{1}^{\prime \prime \prime}(t-\tau)\right|\left|f_{1}(\tau)\right| d \tau \\
& \leq a_{10} x_{12}+a_{11} x_{11}+2 a_{12}-2 \int_{0}^{t} K_{1}^{\prime \prime \prime}(\tau) d \tau \\
& =a_{10} x_{12}+a_{11} x_{11}+4 a_{12}-2 K_{1}^{\prime \prime}(t) \\
& \leq a_{10} x_{12}+a_{11} x_{11}+4 a_{12} \\
& =\frac{1}{9}\left(7 a_{10}^{3}+57 a_{10} a_{11}+36 a_{12}\right)
\end{aligned}
$$


for all $t$.

Theorem 2. Let us consider the equation

$$
f_{2}(t)=1-\int_{0}^{t} K_{2}(t-\tau) f_{2}(\tau) d \tau=1-K_{2} * f_{2}
$$

If

(1) $K_{2} \in C^{4}[0, \infty), K_{2}(t)>0, K_{2}^{\prime}(t)>0, K_{2}^{\prime \prime}(t)>0, K_{2}^{\prime \prime \prime}(t)>0$ and $K_{2}^{(4)}(t) \leq 0$ for all $t$,

(2) $a_{20}^{2}<3 a_{21}$,

(3) $\frac{8}{3} a_{21} \leq a_{20}^{2}$

(4) $a_{20}^{3}-4 a_{20} a_{21}+8 a_{22} \leq 0$,

(5) $-3 a_{20}^{4}+16 a_{20}^{2} a_{21}-64 a_{20} a_{22}+256 a_{23} \leq 0$,

then the solution of (2.13) satisfies the inequalities

$$
\begin{aligned}
& \left|f_{2}^{\prime}(t)\right| \leq \frac{15 a_{20}}{4},\left|f_{2}^{\prime \prime}(t)\right| \leq \frac{1}{8}\left(-a_{20}^{2}+64 a_{21}\right), \\
& \left|f_{2}^{\prime \prime \prime}(t)\right| \leq \frac{1}{64}\left(-45 a_{20}^{3}+544 a_{20} a_{21}+512 a_{22}\right)
\end{aligned}
$$

and

$$
\left|f_{2}^{(4)}(t)\right| \leq \frac{1}{64}\left(-45 a_{20}^{4}+536 a_{20}^{2} a_{21}+752 a_{20} a_{22}+512 a_{21}^{2}+512 a_{23}\right)
$$

for all $t$, where $a_{20}=K_{2}(0), a_{21}=K_{2}^{\prime}(0), a_{22}=K_{2}^{\prime \prime}(0)$ and $a_{23}=K_{2}^{\prime \prime \prime}(0)$.

Proof. Firstly, it is immediate by Theorem 3.3 of [6] that $f_{2}$ provides the inequality $\left|f_{2}(t)\right| \leq 4$ for all $t$. By choosing the function $g$ and the number $\gamma$ as $g(t)=e^{-\gamma t}$ and $\gamma=\gamma_{2}=a_{20} / 4$ in the Equivalence Theorem, we derive the equivalent equation to (2.13) of the form

$$
f_{2}(t)=e^{-\gamma t}-\int_{0}^{t} L_{2}(t-\tau) f_{2}(\tau) d \tau=e^{-\gamma t}-L_{2} * f_{2},
$$

where

$$
L_{2}(t)=\left(a_{20}-\gamma\right) e^{-\gamma t}+K_{2}^{\prime} * e^{-\gamma t} .
$$


Thus,

$$
\begin{aligned}
& L_{2}^{\prime}(t)=\left(\gamma^{2}-a_{20} \gamma+a_{21}\right) e^{-\gamma t}+K_{2}^{\prime \prime} * e^{-\gamma t} \\
& L_{2}^{\prime \prime}(t)=\left(-\gamma^{3}+a_{20} \gamma^{2}-a_{21} \gamma+a_{22}\right) e^{-\gamma t}+K_{2}^{\prime \prime \prime} * e^{-\gamma t}
\end{aligned}
$$

and

$$
L_{2}^{\prime \prime \prime}(t)=\left(\gamma^{4}-a_{20} \gamma^{3}+a_{21} \gamma^{2}-a_{22} \gamma+a_{23}\right) e^{-\gamma t}+K_{2}^{(4)} * e^{-\gamma t} .
$$

It is known that $L_{2}$ satisfies all of the conditions of Theorem 1 from the proof of Theorem 3.3 in [6]. Hence, the first three derivatives of the solution of the equation $h_{2}(t)=1-L_{2} * h_{2}$ must verify the inequalities

$$
\begin{aligned}
\left|h_{2}^{\prime}(t)\right| & \leq \frac{7 L_{2}(0)}{3}=\frac{7\left(a_{20}-\gamma\right)}{3}=\frac{7 a_{20}}{4}=y_{21}, \\
\left|h_{2}^{\prime \prime}(t)\right| & \leq \frac{1}{9}\left[7\left(L_{2}(0)\right)^{2}+36 L_{2}^{\prime}(0)\right] \\
& =\frac{1}{9}\left[7\left(a_{20}-\gamma\right)^{2}+36\left(\gamma^{2}-a_{20} \gamma+a_{21}\right)\right] \\
& =\frac{1}{16}\left(-5 a_{20}^{2}+64 a_{21}\right)=y_{22}
\end{aligned}
$$

and

$$
\begin{aligned}
\left|h_{2}^{\prime \prime \prime}(t)\right| \leq & \frac{1}{9}\left[7\left(L_{2}(0)\right)^{3}+57 L_{2}(0) L_{2}^{\prime}(0)+36 L_{2}^{\prime \prime}(0)\right] \\
= & \frac{1}{9}\left[7\left(a_{20}-\gamma\right)^{3}+57\left(a_{20}-\gamma\right)\left(\gamma^{2}-a_{20} \gamma+a_{21}\right)+\right. \\
& \left.+36\left(-\gamma^{3}+a_{20} \gamma^{2}-a_{21} \gamma+a_{22}\right)\right] \\
= & \frac{1}{8}\left(-3 a_{20}^{3}+30 a_{20} a_{21}+32 a_{22}\right)=y_{23}
\end{aligned}
$$

for all $t$ from Theorem 1. By the Convolution Theorem, the solution $f_{2}$ of the equivalent equation (2.14) can be written by means of $h_{2}$ of the form $f_{2}(t)=h_{2}(t)-\gamma h_{2} * e^{-\gamma t}$. Thus

$$
\begin{aligned}
f_{2}^{\prime}(t) & =h_{2}^{\prime}(t)-\gamma e^{-\gamma t}-\gamma h_{2}^{\prime} * e^{-\gamma t} \\
f_{2}^{\prime \prime}(t) & =h_{2}^{\prime \prime}(t)+\gamma^{2} e^{-\gamma t}-\gamma h_{2}^{\prime}(0) e^{-\gamma t}-\gamma h_{2}^{\prime \prime} * e^{-\gamma t}
\end{aligned}
$$

and

$$
f_{2}^{\prime \prime \prime}(t)=h_{2}^{\prime \prime \prime}(t)-\gamma^{3} e^{-\gamma t}+\gamma^{2} h_{2}^{\prime}(0) e^{-\gamma t}-\gamma h_{2}^{\prime \prime}(0) e^{-\gamma t}-\gamma h_{2}^{\prime \prime \prime} * e^{-\gamma t} .
$$


Hence, the inequalities

$$
\begin{gathered}
\left|f_{2}^{\prime}(t)\right| \leq\left|h_{2}^{\prime}(t)\right|+\gamma+\gamma \int_{0}^{t}\left|h_{2}^{\prime}(t-\tau)\right| e^{-\gamma \tau} d \tau \\
\leq y_{21}+\gamma+\gamma y_{21} \int_{0}^{t} e^{-\gamma \tau} d \tau=y_{21}+\gamma+y_{21}-y_{21} e^{-\gamma t} \\
=2 y_{21}+\gamma-y_{21} e^{-\gamma t} \leq 2 y_{21}+\gamma=\frac{15 a_{20}}{4}=x_{21}, \\
\left|f_{2}^{\prime \prime}(t)\right| \leq\left|h_{2}^{\prime \prime}(t)\right|+\gamma^{2}+\gamma\left|h_{2}^{\prime}(0)\right|+\gamma \int_{0}^{t}\left|h_{2}^{\prime \prime}(t-\tau)\right| e^{-\gamma \tau} d \tau \\
\leq y_{22}+\gamma^{2}+\gamma y_{21}+\gamma y_{22} \int_{0}^{t} e^{-\gamma \tau} d \tau=2 y_{22}+\gamma^{2}+\gamma y_{21}-y_{22} e^{-\gamma t} \\
\leq 2 y_{22}+\gamma^{2}+\gamma y_{21}=\frac{1}{8}\left(-a_{20}^{2}+64 a_{21}\right)=x_{22}
\end{gathered}
$$

and

$$
\begin{aligned}
\left|f_{2}^{\prime \prime \prime}(t)\right| \leq & \left|h_{2}^{\prime \prime \prime}(t)\right|+\gamma^{3}+\gamma^{2}\left|h_{2}^{\prime}(0)\right|+\gamma\left|h_{2}^{\prime \prime}(0)\right|+ \\
& +\gamma \int_{0}^{t}\left|h_{2}^{\prime \prime \prime}(t-\tau)\right| e^{-\gamma \tau} d \tau \\
\leq & y_{23}+\gamma^{3}+\gamma^{2} y_{21}+\gamma y_{22}+\gamma y_{23} \int_{0}^{t} e^{-\gamma \tau} d \tau \\
= & 2 y_{23}+\gamma^{3}+\gamma^{2} y_{21}+\gamma y_{22}-y_{23} e^{-\gamma t} \\
\leq & 2 y_{23}+\gamma^{3}+\gamma^{2} y_{21}+\gamma y_{22} \\
= & \frac{1}{64}\left(-45 a_{20}^{3}+544 a_{20} a_{21}+512 a_{22}\right)=b_{23}
\end{aligned}
$$

are obtained for all $t$ and $\gamma=\gamma_{2}=a_{20} / 4$.

On the other hand, differentiation of the equivalent equation (2.14) leads to

$$
f_{2}^{\prime \prime \prime}(t)=-\gamma^{3} e^{-\gamma t}-L_{2}(0) f_{2}^{\prime \prime}(t)-L_{2}^{\prime}(0) f_{2}^{\prime}(t)-L_{2}^{\prime \prime}(0) f_{2}(t)-L_{2}^{\prime \prime \prime} * f_{2} .
$$


Taking $\gamma=\gamma_{2}=a_{20} / 4$, the inequality

$$
\begin{aligned}
\left|f_{2}^{\prime \prime \prime}(t)\right| \leq & \gamma^{3}+L_{2}(0)\left|f_{2}^{\prime \prime}(t)\right|+L_{2}^{\prime}(0)\left|f_{2}^{\prime}(t)\right|+ \\
& +L_{2}^{\prime \prime}(0)\left|f_{2}(t)\right|+\int_{0}^{t}\left|L_{2}^{\prime \prime \prime}(t-\tau)\right|\left|f_{2}(\tau)\right| d \tau \\
\leq & \gamma^{3}+\left(a_{20}-\gamma\right) x_{22}+\left(\gamma^{2}-a_{20} \gamma+a_{21}\right) x_{21}+ \\
& +4\left(-\gamma^{3}+a_{20} \gamma^{2}-a_{21} \gamma+a_{22}\right)-4 \int_{0}^{t} L_{2}^{\prime \prime \prime}(\tau) d \tau \\
= & \gamma^{3}+\left(a_{20}-\gamma\right) x_{22}+\left(\gamma^{2}-a_{20} \gamma+a_{21}\right) x_{21}+ \\
& +8\left(-\gamma^{3}+a_{20} \gamma^{2}-a_{21} \gamma+a_{22}\right)-4 L_{2}^{\prime \prime}(t) \\
\leq & \gamma^{3}+\left(a_{20}-\gamma\right) x_{22}+\left(\gamma^{2}-a_{20} \gamma+a_{21}\right) x_{21}+ \\
& +8\left(-\gamma^{3}+a_{20} \gamma^{2}-a_{21} \gamma+a_{22}\right) \\
= & \frac{1}{64}\left(-26 a_{20}^{3}+496 a_{20} a_{21}+512 a_{22}\right)=c_{23}
\end{aligned}
$$

is obtained for all $t$.

From condition (3) of Theorem 2,

$$
b_{23}-c_{23}=\frac{a_{20}\left(48 a_{21}-19 a_{20}^{2}\right)}{64} \leq 0
$$

and so by (2.15) and (2.16), we obtain the inequality

$$
\left|f_{2}^{\prime \prime \prime}(t)\right| \leq \min \left\{b_{23}, c_{23}\right\}=b_{23}=\frac{-45 a_{20}^{3}+544 a_{20} a_{21}+512 a_{22}}{64}=x_{23}
$$

for all $t$. From (2.13),

$$
f_{2}^{(4)}(t)=-a_{20} f_{2}^{\prime \prime \prime}(t)-a_{21} f_{2}^{\prime \prime}(t)-a_{22} f_{2}^{\prime}(t)-a_{23} f_{2}(t)-K_{2}^{(4)} * f_{2} .
$$

By using the inequalities obtained above and $\left|f_{2}(t)\right| \leq 4$, the bound of $f_{2}^{(4)}$ is found as

$$
\begin{aligned}
\left|f_{2}^{(4)}(t)\right| \leq & a_{20}\left|f_{2}^{\prime \prime \prime}(t)\right|+a_{21}\left|f_{2}^{\prime \prime}(t)\right|+a_{22}\left|f_{2}^{\prime}(t)\right|+a_{23}\left|f_{2}(t)\right|+ \\
& +\int_{0}^{t}\left|K_{2}^{(4)}(t-\tau)\right|\left|f_{2}(\tau)\right| d \tau \\
\leq & a_{20} x_{23}+a_{21} x_{22}+a_{22} x_{21}+4 a_{23}-4 \int_{0}^{t} K_{2}^{(4)}(\tau) d \tau \\
= & a_{20} x_{23}+a_{21} x_{22}+a_{22} x_{21}+8 a_{23}-4 K_{2}^{\prime \prime \prime}(t) \\
\leq & a_{20} x_{23}+a_{21} x_{22}+a_{22} x_{21}+8 a_{23} \\
= & \frac{1}{64}\left(-45 a_{20}^{4}+536 a_{20}^{2} a_{21}+752 a_{20} a_{22}+512 a_{21}^{2}+512 a_{23}\right)
\end{aligned}
$$

for all $t$ which completes the proof. 
Theorem 3. Let us consider the equation

$$
f_{3}(t)=1-\int_{0}^{t} K_{3}(t-\tau) f_{3}(\tau) d \tau=1-K_{3} * f_{3} .
$$

If

(1) $K_{3} \in C^{5}[0, \infty), K_{3}(t)>0, K_{3}^{\prime}(t)>0, \ldots, K_{3}^{(4)}(t)>0$ and $K_{3}^{(5)}(t) \leq 0$ for all $t$,

(2) $a_{30}^{2}<\frac{8}{3} a_{31}$

(3) $\frac{5}{2} a_{31} \leq a_{30}^{2}$,

(4) $4 a_{30}^{3}-15 a_{30} a_{31}+25 a_{32} \leq 0$,

(5) $-3 a_{30}^{4}+15 a_{30}^{2} a_{31}-50 a_{30} a_{32}+125 a_{33} \leq 0$,

(6) $4 a_{30}^{3}-25 a_{30} a_{31}+125 a_{32}>0$,

(7) $4 a_{30}^{5}-25 a_{30}^{3} a_{31}+125 a_{30}^{2} a_{32}-625 a_{30} a_{33}+3125 a_{34} \leq 0$,

then

$$
\begin{gathered}
\left|f_{3}^{\prime}(t)\right| \leq \frac{31 a_{30}}{5} \\
\left|f_{3}^{\prime \prime}(t)\right| \leq \frac{1}{25}\left(-52 a_{30}^{2}+400 a_{31}\right) \\
\left|f_{3}^{\prime \prime \prime}(t)\right| \leq \frac{1}{125}\left(-316 a_{30}^{3}+1500 a_{30} a_{31}+2000 a_{32}\right) \\
\left|f_{3}^{(4)}(t)\right| \leq \frac{1}{625}\left(-1031 a_{30}^{4}+2400 a_{30}^{2} a_{31}+10750 a_{30} a_{32}+\right. \\
\left.+10000 a_{31}^{2}+10000 a_{33}\right)
\end{gathered}
$$

and

$$
\begin{aligned}
\left|f_{3}^{(5)}(t)\right| \leq & \frac{1}{625}\left(-1031 a_{30}^{5}+820 a_{30}^{3} a_{31}+17500 a_{30} a_{31}^{2}+9450 a_{30}^{2} a_{32}+\right. \\
& \left.+13875 a_{30} a_{33}+20000 a_{31} a_{32}+10000 a_{34}\right)
\end{aligned}
$$

for all $t$, where $a_{30}=K_{3}(0), a_{31}=K_{3}^{\prime}(0), a_{32}=K_{3}^{\prime \prime}(0), a_{33}=K_{3}^{\prime \prime \prime}(0)$ and $a_{34}=K_{3}^{(4)}(0)$. 
Proof. It is immediate by Theorem 3 of [8] that the inequality $\left|f_{3}(t)\right| \leq 8$ is held for all $t$. If the function $g$ is chosen as $g(t)=e^{-\gamma t}\left(\gamma=\gamma_{3}=a_{30} / 5\right)$ in the Equivalence Theorem, we get the equivalent equation to (2.17) as

$$
f_{3}(t)=e^{-\gamma t}-\int_{0}^{t} L_{3}(t-\tau) f_{3}(\tau) d \tau=e^{-\gamma t}-L_{3} * f_{3},
$$

where

$$
L_{3}(t)=\left(a_{30}-\gamma\right) e^{-\gamma t}+K_{3}^{\prime} * e^{-\gamma t}
$$

Thus,

$$
\begin{aligned}
L_{3}^{\prime}(t) & =\left(\gamma^{2}-a_{30} \gamma+a_{31}\right) e^{-\gamma t}+K_{3}^{\prime \prime} * e^{-\gamma t}, \\
L_{3}^{\prime \prime}(t) & =\left(-\gamma^{3}+a_{30} \gamma^{2}-a_{31} \gamma+a_{32}\right) e^{-\gamma t}+K_{3}^{\prime \prime \prime} * e^{-\gamma t}, \\
L_{3}^{\prime \prime \prime}(t) & =\left(\gamma^{4}-a_{30} \gamma^{3}+a_{31} \gamma^{2}-a_{32} \gamma+a_{33}\right) e^{-\gamma t}+K_{3}^{(4)} * e^{-\gamma t}
\end{aligned}
$$

and

$$
L_{3}^{(4)}(t)=\left(-\gamma^{5}+a_{30} \gamma^{4}-a_{31} \gamma^{3}+a_{32} \gamma^{2}-a_{33} \gamma+a_{34}\right) e^{-\gamma t}+K_{3}^{(5)} * e^{-\gamma t} .
$$

It is known that $L_{3}$ satisfies all of the conditions of Theorem 2 by the proof of Theorem 3 in [8]. Hence, the first four derivatives of the solution of the equation $h_{3}(t)=1-L_{3} * h_{3}$ verify the inequalities

$$
\begin{gathered}
\left|h_{3}^{\prime}(t)\right| \leq \frac{15 L_{3}(0)}{4}=3 a_{30}, \\
\left|h_{3}^{\prime \prime}(t)\right| \leq \frac{1}{8}\left[-\left(L_{3}(0)\right)^{2}+64 L_{3}^{\prime}(0)\right]=\frac{1}{25}\left(-34 a_{30}^{2}+200 a_{31}\right), \\
\left|h_{3}^{\prime \prime \prime}(t)\right| \leq \frac{1}{64}\left[-45\left(L_{3}(0)\right)^{3}+544 L_{3}(0) L_{3}^{\prime}(0)+512 L_{3}^{\prime \prime}(0)\right] \\
=\frac{1}{125}\left(-149 a_{30}^{3}+650 a_{30} a_{31}+1000 a_{32}\right)
\end{gathered}
$$

and

$$
\begin{aligned}
\left|h_{3}^{(4)}(t)\right| \leq & \frac{1}{64}\left[-45\left(L_{3}(0)\right)^{4}+536\left(L_{3}(0)\right)^{2} L_{3}^{\prime}(0)+752 L_{3}(0) L_{3}^{\prime \prime}(0)+\right. \\
& \left.+512\left(L_{3}^{\prime}(0)\right)^{2}+512 L_{3}^{\prime \prime \prime}(0)\right] \\
= & \frac{1}{625}\left(-432 a_{30}^{4}+775 a_{30}^{2} a_{31}+4875 a_{30} a_{32}+5000 a_{31}^{2}+5000 a_{33}\right)
\end{aligned}
$$


for all $t$ and $\gamma=\gamma_{3}$ from Theorem 2. By the Convolution Theorem, the solution $f_{3}$ of the equivalent equation (2.23) can be written by means of $h_{3}$ of the form $f_{3}(t)=h_{3}(t)-\gamma h_{3} * e^{-\gamma t}$. Thus,

$$
\begin{aligned}
f_{3}^{\prime}(t) & =h_{3}^{\prime}(t)-\gamma e^{-\gamma t}-\gamma h_{3}^{\prime} * e^{-\gamma t} \\
f_{3}^{\prime \prime}(t) & =h_{3}^{\prime \prime}(t)+\gamma^{2} e^{-\gamma t}-\gamma h_{3}^{\prime}(0) e^{-\gamma t}-\gamma h_{3}^{\prime \prime} * e^{-\gamma t} \\
f_{3}^{\prime \prime \prime}(t) & =h_{3}^{\prime \prime \prime}(t)-\gamma^{3} e^{-\gamma t}+\gamma^{2} h_{3}^{\prime}(0) e^{-\gamma t}-\gamma h_{3}^{\prime \prime}(0) e^{-\gamma t}-\gamma h_{3}^{\prime \prime \prime} * e^{-\gamma t}
\end{aligned}
$$

and

$$
\begin{aligned}
f_{3}^{(4)}(t)= & h_{3}^{(4)}(t)+\gamma^{4} e^{-\gamma t}-\gamma^{3} h_{3}^{\prime}(0) e^{-\gamma t}+\gamma^{2} h_{3}^{\prime \prime}(0) e^{-\gamma t}-\gamma h_{3}^{\prime \prime \prime}(0) e^{-\gamma t}- \\
& -\gamma h_{3}^{(4)} * e^{-\gamma t} .
\end{aligned}
$$

By taking $\gamma=\gamma_{3}=a_{30} / 5$, inequalities (2.18), (2.19), (2.20) and

$$
\begin{aligned}
\left|f_{3}^{(4)}(t)\right| \leq & \frac{1}{625}\left(-1031 a_{30}^{4}+2400 a_{30}^{2} a_{31}+10000 a_{31}^{2}+\right. \\
& \left.+10750 a_{30} a_{32}+10000 a_{33}\right) \\
= & b_{34}
\end{aligned}
$$

are obtained for all $t$. On the other hand, differentiation of the equivalent equation (2.23) leads to

$f_{3}^{(4)}(t)=\gamma^{4} e^{-\gamma t}-L_{3}(0) f_{3}^{\prime \prime \prime}(t)-L_{3}^{\prime}(0) f_{3}^{\prime \prime}(t)-L_{3}^{\prime \prime}(0) f_{3}^{\prime}(t)-L_{3}^{\prime \prime \prime}(0) f_{3}(t)-L_{3}^{(4)} * f_{3}$.

Taking $\gamma=\gamma_{3}=a_{30} / 5$, the inequality

$$
\begin{aligned}
\left|f_{3}^{(4)}(t)\right| \leq & \frac{1}{625}\left(-995 a_{30}^{4}+2725 a_{30}^{2} a_{31}+9875 a_{30} a_{32}+\right. \\
& \left.+10000 a_{31}^{2}+10000 a_{33}\right) \\
= & c_{34}
\end{aligned}
$$

is obtained for all $t$ from (2.18), (2.19), (2.20) and $\left|f_{3}(t)\right| \leq 8$. Thus, from (2.24) and (2.25), we have (2.21) for all $t$ by conditions (4) and (3) of Theorem 3. From (2.17),

$$
f_{3}^{(5)}(t)=-a_{30} f_{3}^{(4)}(t)-a_{31} f_{3}^{\prime \prime \prime}(t)-a_{32} f_{3}^{\prime \prime}(t)-a_{33} f_{3}^{\prime}(t)-a_{34} f_{3}(t)-K_{3}^{(5)} * f_{3} .
$$

By inequalities (2.18), (2.19), (2.20), (2.21) obtained above and $\left|f_{3}(t)\right| \leq 8$, we get (2.22) for all $t$ and this concludes the proof of Theorem 3 .

Theorem 4. Consider the equation

$$
f_{4}(t)=1-\int_{0}^{t} K_{4}(t-\tau) f_{4}(\tau) d \tau=1-K_{4} * f_{4}
$$


(1) $K_{4} \in C^{6}[0, \infty), K_{4}(t)>0, K_{4}^{\prime}(t)>0, \ldots, K_{4}^{(5)}(t)>0$ and $K_{4}^{(6)}(t) \leq 0$ for all $t$,

(2) $a_{40}^{2}<\frac{5}{2} a_{41}$

(3) $\frac{12}{5} a_{41} \leq a_{40}^{2}$,

(4) $5 a_{40}^{3}-18 a_{40} a_{41}+27 a_{42} \leq 0$,

(5) $-5 a_{40}^{4}+24_{40}^{2} a_{41}-72 a_{40} a_{42}+144 a_{43} \leq 0$,

(6) $7 a_{40}^{3}-36 a_{40} a_{41}+108 a_{42}>0$,

(7) $a_{40}^{5}-6 a_{40}^{3} a_{41}+27 a_{40}^{2} a_{42}-108 a_{40} a_{43}+324 a_{44} \leq 0$,

(8) $5 a_{40}^{3}-36 a_{40} a_{41}+216 a_{42}>0$,

(9) $-5 a_{40}^{4}+36 a_{40}^{2} a_{41}-216 a_{40} a_{42}+1296 a_{43}>0$,

(10) $-5 a_{40}^{6}+36 a_{40}^{4} a_{41}-216 a_{40}^{3} a_{42}+1296 a_{40}^{2} a_{43}-7776 a_{40} a_{44}+46656 a_{45} \leq 0$, then

$$
\begin{gathered}
\left|f_{4}^{\prime}(t)\right| \leq \frac{21 a_{40}}{2}, \\
\left|f_{4}^{\prime \prime}(t)\right| \leq \frac{1}{9}\left(-58 a_{40}^{2}+288 a_{41}\right), \\
\left|f_{4}^{\prime \prime \prime}(t)\right| \leq \frac{1}{54}\left(-293 a_{40}^{3}+936 a_{40} a_{41}+1728 a_{42}\right), \\
\left|f_{4}^{(4)}(t)\right| \leq \frac{1}{648}\left(-1079 a_{40}^{4}-3744 a_{40}^{2} a_{41}+20736 a_{41}^{2}+\right. \\
\left.\quad+16848 a_{40} a_{42}+20736 a_{43}\right), \\
\left|f_{4}^{(5)}(t)\right| \leq \frac{1}{7776}\left(-5117 a_{40}^{5}-88992 a_{40}^{3} a_{41}+300672 a_{40} a_{41}^{2}+71712 a_{40}^{2} a_{42}+\right. \\
\left.+266976 a_{40} a_{43}+497664 a_{41} a_{42}+248832 a_{44}\right)
\end{gathered}
$$

and

$$
\begin{aligned}
\left|f_{4}^{(6)}(t)\right| \leq & \frac{1}{7776}\left(-5117 a_{40}^{6}-101940 a_{40}^{4} a_{41}+255744 a_{40}^{2} a_{41}^{2}+29520 a_{40}^{3} a_{42}+\right. \\
& +216864 a_{40}^{2} a_{43}+834624 a_{40} a_{41} a_{42}+330480 a_{40} a_{44}+248832 a_{41}^{3}+ \\
& \left.+497664 a_{41} a_{43}+248832 a_{42}^{2}+248832 a_{45}\right)
\end{aligned}
$$

for all $t$, where $a_{40}=K_{4}(0), a_{41}=K_{4}^{\prime}(0), a_{42}=K_{4}^{\prime \prime}(0), a_{43}=K_{4}^{\prime \prime \prime}(0)$, $a_{44}=K_{4}^{(4)}(0)$ and $a_{45}=K_{4}^{(5)}(0)$. 
Proof. It is easily seen by Theorem 4 of [8] that $f_{4}$ satisfies the inequality $\left|f_{4}(t)\right| \leq 16$ for all $t$. By choosing $g(t)=e^{-\gamma t}$ and $\gamma=\gamma_{4}=a_{40} / 6$ in the Equivalence Theorem, we get the equivalent equation to (2.26) as

$$
f_{4}(t)=e^{-\gamma t}-\int_{0}^{t} L_{4}(t-\tau) f_{4}(\tau) d \tau=e^{-\gamma t}-L_{4} * f_{4},
$$

where

$$
L_{4}(t)=\left(a_{40}-\gamma\right) e^{-\gamma t}+K_{4}^{\prime} * e^{-\gamma t}
$$

Thus,

$$
\begin{aligned}
L_{4}^{\prime}(t) & =\left(\gamma^{2}-a_{40} \gamma+a_{41}\right) e^{-\gamma t}+K_{4}^{\prime \prime} * e^{-\gamma t} \\
L_{4}^{\prime \prime}(t) & =\left(-\gamma^{3}+a_{40} \gamma^{2}-a_{41} \gamma+a_{42}\right) e^{-\gamma t}+K_{4}^{\prime \prime \prime} * e^{-\gamma t} \\
L_{4}^{\prime \prime \prime}(t) & =\left(\gamma^{4}-a_{40} \gamma^{3}+a_{41} \gamma^{2}-a_{42} \gamma+a_{43}\right) e^{-\gamma t}+K_{4}^{(4)} * e^{-\gamma t} \\
L_{4}^{(4)}(t) & =\left(-\gamma^{5}+a_{40} \gamma^{4}-a_{41} \gamma^{3}+a_{42} \gamma^{2}-a_{43} \gamma+a_{44}\right) e^{-\gamma t}+K_{4}^{(5)} * e^{-\gamma t}
\end{aligned}
$$

and

$L_{4}^{(5)}(t)=\left(\gamma^{6}-a_{40} \gamma^{5}+a_{41} \gamma^{4}-a_{42} \gamma^{3}+a_{43} \gamma^{2}-a_{44} \gamma+a_{45}\right) e^{-\gamma t}+K_{4}^{(6)} * e^{-\gamma t}$.

It is known that $L_{4}$ satisfies all of the conditions of Theorem 3 from the proof of Theorem 4 in [8]. Hence, the first five derivatives of the solution of the equation $h_{4}(t)=1-L_{4} * h_{4}$ verify the inequalities

$$
\begin{gathered}
\left|h_{4}^{\prime}(t)\right| \leq \frac{31 L_{4}(0)}{5}=\frac{31 a_{40}}{6}, \\
\left|h_{4}^{\prime \prime}(t)\right| \leq \frac{1}{25}\left[-52\left(L_{4}(0)\right)^{2}+400 L_{4}^{\prime}(0)\right]=\frac{1}{3}\left(-11 a_{40}^{2}+48 a_{41}\right), \\
\left|h_{4}^{\prime \prime \prime}(t)\right| \leq \frac{1}{125}\left[-316\left(L_{4}(0)\right)^{3}+1500 L_{4}(0) L_{4}^{\prime}(0)+2000 L_{4}^{\prime \prime}(0)\right] \\
\left|h_{4}^{(4)}(t)\right| \leq \frac{1}{27}\left(-67 a_{40}^{3}+198 a_{40} a_{41}+432 a_{42}\right), \\
+\frac{1}{625}\left[-1031\left(L_{4}(0)\right)^{4}+2400\left(L_{4}(0)\right)^{2} L_{4}^{\prime}(0)+\right. \\
=\frac{1}{1296}\left(-761 a_{40}^{4}-4824 a_{40}^{2} a_{41}+15120 a_{40} a_{42}+\right. \\
\left.+20736 a_{41}^{2}+20736 a_{43}\right)
\end{gathered}
$$


and

$$
\begin{aligned}
\left|h_{4}^{(5)}(t)\right| \leq & \frac{1}{625}\left[-1031\left(L_{4}(0)\right)^{5}+820\left(L_{4}(0)\right)^{3} L_{4}^{\prime}(0)+17500 L_{4}(0)\left(L_{4}^{\prime}(0)\right)^{2}+\right. \\
& +9450\left(L_{4}(0)\right)^{2} L_{4}^{\prime \prime}(0)+13875 L_{4}(0) L_{4}^{\prime \prime \prime}(0)+20000 L_{4}^{\prime}(0) L_{4}^{\prime \prime}(0)+ \\
& \left.+10000 L_{4}^{(4)}(0)\right] \\
= & \frac{1}{648}\left(-155 a_{40}^{5}-3597 a_{40}^{3} a_{41}+11664 a_{40} a_{41}^{2}+2214 a_{40}^{2} a_{42}+\right. \\
& \left.+10260 a_{40} a_{43}+20736 a_{41} a_{42}+10368 a_{44}\right)
\end{aligned}
$$

for all $t$ and $\gamma=\gamma_{4}$ by Theorem 3. By the Convolution Theorem, the solution $f_{4}$ of the equivalent equation (2.33) can be written by means of $h_{4}$ as $f_{4}(t)=h_{4}(t)-\gamma h_{4} * e^{-\gamma t}$. Thus,

$$
\begin{aligned}
f_{4}^{\prime}(t)= & h_{4}^{\prime}(t)-\gamma e^{-\gamma t}-\gamma h_{4}^{\prime} * e^{-\gamma t} \\
f_{4}^{\prime \prime}(t)= & h_{4}^{\prime \prime}(t)+\gamma^{2} e^{-\gamma t}-\gamma h_{4}^{\prime}(0) e^{-\gamma t}-\gamma h_{4}^{\prime \prime} * e^{-\gamma t} \\
f_{4}^{\prime \prime \prime}(t)= & h_{4}^{\prime \prime \prime}(t)-\gamma^{3} e^{-\gamma t}+\gamma^{2} h_{4}^{\prime}(0) e^{-\gamma t}-\gamma h_{4}^{\prime \prime}(0) e^{-\gamma t}-\gamma h_{4}^{\prime \prime \prime} * e^{-\gamma t} \\
f_{4}^{(4)}(t)= & h_{4}^{(4)}(t)+\gamma^{4} e^{-\gamma t}-\gamma^{3} h_{4}^{\prime}(0) e^{-\gamma t}+\gamma^{2} h_{4}^{\prime \prime}(0) e^{-\gamma t}-\gamma h_{4}^{\prime \prime \prime}(0) e^{-\gamma t}- \\
& -\gamma h_{4}^{(4)} * e^{-\gamma t}
\end{aligned}
$$

and

$$
\begin{aligned}
f_{4}^{(5)}(t)= & h_{4}^{(5)}(t)-\gamma^{5} e^{-\gamma t}+\gamma^{4} h_{4}^{\prime}(0) e^{-\gamma t}-\gamma^{3} h_{4}^{\prime \prime}(0) e^{-\gamma t}+ \\
& +\gamma^{2} h_{4}^{\prime \prime \prime}(0) e^{-\gamma t}-\gamma h_{4}^{(4)}(0) e^{-\gamma t}-\gamma h_{4}^{(5)} * e^{-\gamma t} .
\end{aligned}
$$

Therefore, by taking $\gamma=\gamma_{4}=a_{40} / 5$, inequalities (2.27), (2.28), (2.29), (2.30) and

$$
\begin{aligned}
\left|f_{4}^{(5)}(t)\right| \leq & \frac{1}{7776}\left(-5117 a_{40}^{5}-88992 a_{40}^{3} a_{41}+300672 a_{40} a_{41}^{2}+71712 a_{40}^{2} a_{42}+\right. \\
& \left.+266976 a_{40} a_{43}+497664 a_{41} a_{42}+248832 a_{44}\right) \\
= & b_{45}
\end{aligned}
$$

are obtained for all $t$.

On the other hand, differentiation of the equivalent equation (2.33) leads to

$$
\begin{aligned}
f_{4}^{(5)}(t)= & -\gamma^{5} e^{-\gamma t}-L_{4}(0) f_{4}^{(4)}(t)-L_{4}^{\prime}(0) f_{4}^{\prime \prime \prime}(t)-L_{4}^{\prime \prime}(0) f_{4}^{\prime \prime}(t)- \\
& -L_{4}^{\prime \prime \prime}(0) f_{4}^{\prime}(t)-L_{4}^{(4)}(0) f_{4}(t)-L_{4}^{(5)} * f_{4} .
\end{aligned}
$$

Taking $\gamma=\gamma_{4}=a_{40} / 5$, the inequality

$$
\begin{aligned}
\left|f_{4}^{(5)}(t)\right| \leq & \frac{1}{7776}\left(-6244 a_{40}^{5}-83124 a_{40}^{3} a_{41}+300672 a_{40} a_{41}^{2}+77112 a_{40}^{2} a_{42}+\right. \\
& \left.+247536 a_{40} a_{43}+497664 a_{41} a_{42}+248832 a_{44}\right) \\
= & c_{45}
\end{aligned}
$$


is obtained for all $t$ from inequalities (2.27), (2.28), (2.29), (2.30) and $\left|f_{4}(t)\right| \leq$ 16. Hence, we have by (2.34) and (2.35) that inequality (2.31) holds for all $t$ from conditions (5), (4) and (2) of Theorem 4.

Finally, from (2.26), we get

$$
\begin{aligned}
f_{4}^{(6)}(t)= & -a_{40} f_{4}^{(5)}(t)-a_{41} f_{4}^{(4)}(t)-a_{42} f_{4}^{\prime \prime \prime}(t)-a_{43} f_{4}^{\prime \prime}(t)-a_{44} f_{4}^{\prime}(t)- \\
& -a_{45} f_{4}(t)-K_{4}^{(6)} * f_{4} .
\end{aligned}
$$

By inequalities (2.27), (2.28), (2.29), (2.30), (2.31) obtained above and $\left|f_{4}(t)\right| \leq$ 16 , we derive inequality (2.32) for all $t$ and this completes the proof of Theorem 4.

By continuing this process for $n \in \mathbb{N}_{2}$, we have Theorem $n$ which can be stated as follows:

Theorem n. Let us consider the equation

$$
f_{n}(t)=1-\int_{0}^{t} K_{n}(t-\tau) f_{n}(\tau) d \tau=1-K_{n} * f_{n}
$$

under the following assumptions:

(1) $K_{n} \in C^{n+2}[0, \infty), K_{n}(t)>0, K_{n}^{\prime}(t)>0, \ldots, K_{n}^{(n+1)}(t)>0$ and $K_{n}^{(n+2)}(t) \leq 0$ for all $t$

(2) $a_{n 0}^{2}<\frac{2(n+1)}{n} a_{n 1}$ and

(3) $\frac{2(n+2)}{n+1} a_{n 1} \leq a_{n 0}^{2}$.

Furthermore, conditions (4) th, (5) th,..., $\left[4+\frac{(n-2)(n-1)}{2}\right]$ th of Theorem $n$ are the inequalities obtained by taking $\left(a_{n 0}-\gamma_{n}\right),\left(\gamma_{n}^{2}-a_{n 0} \gamma_{n}+a_{n 1}\right), \ldots$, $\left((-1)^{n+1} \gamma_{n}^{n+1}+(-1)^{n} a_{n 0} \gamma_{n}^{n}+(-1)^{n-1} a_{n 1} \gamma_{n}^{n-1}+\cdots-a_{n(n-1)} \gamma_{n}+a_{n n}\right)$, respectively instead of the constants $a_{(n-1) 0}, a_{(n-1) 1}, \ldots, a_{(n-1) n}$ in conditions (4) th, (5) th, ..., $\left[4+\frac{(n-2)(n-1)}{2}\right]$ th of Theorem $(n-1)$ and by writing $\gamma_{n}=\frac{a_{n 0}}{n+2}$.

Moreover, conditions $\left[5+\frac{(n-2)(n-1)}{2}\right]$ th, $\left[6+\frac{(n-2)(n-1)}{2}\right]$ th, .., $\left[5+\frac{n(n-1)-4}{2}\right]$ th and $\left[5+\frac{n(n-1)-2}{2}\right]$ th of Theorem $n$ are also the inequalities $p_{3}\left(\gamma_{n}\right), p_{4}\left(\gamma_{n}\right)$, $\ldots, p_{n}\left(\gamma_{n}\right)>0$ and $p_{n+2}\left(\gamma_{n}\right) \leq 0$, respectively.

Besides, let us define the first constant $x_{n 1}$ as

$$
x_{n 1}=z_{n} a_{n 0},
$$


where

$$
z_{1}=\frac{7}{3}, z_{n}=\frac{2(n+1) z_{n-1}+1}{n+2},(n \geq 2) .
$$

The constants $x_{n 2}, x_{n 3}, \ldots, x_{n(n+2)}$ are defined as follows:

$$
x_{n 2}=2 y_{n 2}+\gamma_{n}^{2}+\gamma_{n} y_{n 1},
$$

where $y_{n 1}$ has been obtained by taking $p_{1}\left(\gamma_{n}\right)$ instead of the constant $a_{(n-1) 0}$ which is included at $x_{(n-1) 1}$ which is the bound of $f_{n-1}^{\prime}$ in Theorem $(n-1)$ and $y_{n 2}$ has been got by taking $p_{1}\left(\gamma_{n}\right), p_{2}\left(\gamma_{n}\right)$, respectively instead of the constants $a_{(n-1) 0}, a_{(n-1) 1}$ which are included at $x_{(n-1) 2}$ which is the bound of $f_{n-1}^{\prime \prime}$ in Theorem $(n-1)$.

$$
x_{n 3}=2 y_{n 3}+\gamma_{n}^{3}+\gamma_{n}^{2} y_{n 1}+\gamma_{n} y_{n 2},
$$

where $y_{n 3}$ has been got by taking $p_{1}\left(\gamma_{n}\right), p_{2}\left(\gamma_{n}\right), p_{3}\left(\gamma_{n}\right)$, respectively instead of the constants $a_{(n-1) 0}, a_{(n-1) 1}, a_{(n-1) 2}$ which are included at $x_{(n-1) 3}$ which is the bound of $f_{n-1}^{\prime \prime \prime}$ in Theorem $(n-1)$.

Similarly,

$$
x_{n n}=2 y_{n n}+\gamma_{n}^{n}+\gamma_{n}^{n-1} y_{n 1}+\gamma_{n}^{n-2} y_{n 2}+\cdots+\gamma_{n} y_{n(n-1)},
$$

where $y_{n n}$ has been obtained by taking $p_{1}\left(\gamma_{n}\right), p_{2}\left(\gamma_{n}\right), \ldots, p_{n}\left(\gamma_{n}\right)$, respectively instead of the constants $a_{(n-1) 0}, a_{(n-1) 1}, \ldots, a_{(n-1)(n-1)}$ which are included at $x_{(n-1) n}$ which is the bound of $f_{n-1}^{(n)}$ in Theorem $(n-1)$.

Additionally, let us define the constant $x_{n(n+1)}$ by

$$
x_{n(n+1)}=\min \left\{b_{n(n+1)}, c_{n(n+1)}\right\}
$$

such that

$$
b_{n(n+1)}=2 y_{n(n+1)}+\gamma_{n}^{n+1}+\gamma_{n}^{n} y_{n 1}+\gamma_{n}^{n-1} y_{n 2}+\cdots+\gamma_{n} y_{n n}
$$

where $y_{n(n+1)}$ has been got by taking $p_{1}\left(\gamma_{n}\right), p_{2}\left(\gamma_{n}\right), \ldots, p_{n+1}\left(\gamma_{n}\right)$, respectively instead of the constants $a_{(n-1) 0}, a_{(n-1) 1}, \ldots, a_{(n-1) n}$ which are included at $x_{(n-1)(n+1)}$ which is the bound of $f_{n-1}^{(n+1)}$ in Theorem $(n-1)$ and

$c_{n(n+1)}=\gamma_{n}^{n+1}+p_{1}\left(\gamma_{n}\right) x_{n n}+p_{2}\left(\gamma_{n}\right) x_{n(n-1)}+\cdots+p_{n}\left(\gamma_{n}\right) x_{n 1}+2^{n+1} p_{n+1}\left(\gamma_{n}\right)$.

Finally, the last constant $x_{n(n+2)}$ is defined by

$$
x_{n(n+2)}=a_{n 0} x_{n(n+1)}+a_{n 1} x_{n n}+\cdots+a_{n n} x_{n 1}+2^{n+1} a_{n(n+1)},
$$


where

$p_{i}(\gamma)=(-1)^{i} \gamma^{i}+(-1)^{i+1} a_{n 0} \gamma^{i-1}+(-1)^{i+2} a_{n 1} \gamma^{i-2}+\cdots-a_{n(i-2)} \gamma+a_{n(i-1)}$

for $i \in\{1,2, \ldots, n+2\}$ and $a_{n 0}=K_{n}(0), a_{n 1}=K_{n}^{\prime}(0), a_{n 2}=K_{n}^{\prime \prime}(0), \ldots$, $a_{n(n+1)}=K_{n}^{(n+1)}(0)$. Then, the solution of equation (2.36) satisfies the inequalities

$\left|f_{n}^{\prime}(t)\right| \leq x_{n 1},\left|f_{n}^{\prime \prime}(t)\right| \leq x_{n 2}, \ldots,\left|f_{n}^{(n+1)}(t)\right| \leq x_{n(n+1)}$ and $\left|f_{n}^{(n+2)}(t)\right| \leq x_{n(n+2)}$

for all $t$ and $n \in \mathbb{N}_{2}$.

Proof. For this, we use the mathematical induction. It is conclude that Theorem $n$ is true for $n=2$. Let us suppose that Theorem $m$ is true. Thus, we observe under the conditions of Theorem $m$ that $\left|f_{m}^{\prime}(t)\right| \leq x_{m 1}$, $\left|f_{m}^{\prime \prime}(t)\right| \leq x_{m 2}, \ldots,\left|f_{m}^{(m+1)}(t)\right| \leq x_{m(m+1)}$ and $\left|f_{m}^{(m+2)}(t)\right| \leq x_{m(m+2)}$ for all $t$. In this case, we try to show that Theorem $n$ is also true for $n=m+1$. Namely, by considering the conditions of Theorem $(m+1)$, we must show that the solution of the equation

$$
f_{m+1}(t)=1-K_{m+1} * f_{m+1}
$$

satisfies also the inequalities $\left|f_{m+1}^{\prime}(t)\right| \leq x_{(m+1) 1},\left|f_{m+1}^{\prime \prime}(t)\right| \leq x_{(m+1) 2}, \ldots$, $\left|f_{m+1}^{(m+2)}(t)\right| \leq x_{(m+1)(m+2)}$ and $\left|f_{m+1}^{(m+3)}(t)\right| \leq x_{(m+1)(m+3)}$ for all $t$. We can immediately assert that $f_{m+1}(t)$ provides the inequality $\left|f_{m+1}(t)\right| \leq 2^{m+1}$ for all $t$ from [8, Theorem $\mathrm{n}]$.

By choosing $\gamma=\gamma_{m+1}=a_{(m+1) 0} /(m+3)$ and $g(t)=e^{-\gamma t}$ in the Equivalence Theorem, we get the equivalent equation to (2.37) of the form

$$
f_{m+1}(t)=e^{-\gamma t}-L_{m+1} * f_{m+1},
$$

where

$$
L_{m+1}(t)=\left(a_{(m+1) 0}-\gamma\right) e^{-\gamma t}+K_{m+1}^{\prime} * e^{-\gamma t} .
$$

Thus,

$$
\begin{aligned}
& L_{m+1}^{\prime}(t)=\left(\gamma^{2}-a_{(m+1) 0} \gamma+a_{(m+1) 1}\right) e^{-\gamma t}+K_{m+1}^{\prime \prime} * e^{-\gamma t} \\
& L_{m+1}^{\prime \prime}(t)=\left(-\gamma^{3}+a_{(m+1) 0} \gamma^{2}-a_{(m+1) 1} \gamma+a_{(m+1) 2}\right) e^{-\gamma t}+K_{m+1}^{\prime \prime \prime} * e^{-\gamma t}
\end{aligned}
$$

and similarly,

$$
\begin{aligned}
L_{m+1}^{(m+1)}(t)= & \left((-1)^{m+2} \gamma^{m+2}+(-1)^{m+1} a_{(m+1) 0} \gamma^{m+1}+(-1)^{m} a_{(m+1) 1} \gamma^{m}+\right. \\
& \left.+\cdots-a_{(m+1) m} \gamma+a_{(m+1)(m+1)}\right) e^{-\gamma t}+K_{m+1}^{(m+2)} * e^{-\gamma t}
\end{aligned}
$$


and

$$
\begin{aligned}
L_{m+1}^{(m+2)}(t)= & \left((-1)^{m+3} \gamma^{m+3}+(-1)^{m+2} a_{(m+1) 0} \gamma^{m+2}+(-1)^{m+1} a_{(m+1) 1} \gamma^{m+1}+\right. \\
& \left.+\cdots-a_{(m+1)(m+1)} \gamma+a_{(m+1)(m+2)}\right) e^{-\gamma t}+K_{m+1}^{(m+3)} * e^{-\gamma t} .
\end{aligned}
$$

It is known that the kernel $L_{m+1}$ of equation (2.38) satisfies all of the conditions of Theorem $m$ by the proof of Theorem $n$ of [8]. Hence, by using Theorem $m$, it is understood that the first derivative of the solution of the equation

$$
h_{m+1}(t)=1-L_{m+1} * h_{m+1}
$$

clearly satisfies the inequality

$\left|h_{m+1}^{\prime}(t)\right| \leq z_{m} L_{m+1}(0)=z_{m}\left(a_{(m+1) 0}-\gamma_{m+1}\right)=\left(\frac{m+2}{m+3}\right) z_{m} a_{(m+1) 0}=y_{(m+1) 1}$

for all $t$. On the other hand, the new constants $y_{(m+1) 2}, y_{(m+1) 3}, \ldots, y_{(m+1)(m+1)}$ are defined as follows:

We derive the constant $y_{(m+1) 2}$ by taking $L_{m+1}(0)=p_{1}\left(\gamma_{m+1}\right)$ and $L_{m+1}^{\prime}(0)=$ $p_{2}\left(\gamma_{m+1}\right)$, respectively instead of the constants $a_{m 0}$ and $a_{m 1}$ in $x_{m 2}$ which is the bound of $f_{m}^{\prime \prime}$ in Theorem $m$. We get the constant $y_{(m+1) 3}$ by taking $L_{m+1}(0)=p_{1}\left(\gamma_{m+1}\right), L_{m+1}^{\prime}(0)=p_{2}\left(\gamma_{m+1}\right)$ and $L_{m+1}^{\prime \prime}(0)=p_{3}\left(\gamma_{m+1}\right)$, respectively instead of the constants $a_{m 0}, a_{m 1}$ and $a_{m 2}$ in $x_{m 3}$ which is the bound of $f_{m}^{\prime \prime \prime}$ in Theorem $m$. Continuing this process, we have the last constant $y_{(m+1)(m+2)}$ by taking $L_{m+1}(0)=p_{1}\left(\gamma_{m+1}\right), L_{m+1}^{\prime}(0)=p_{2}\left(\gamma_{m+1}\right), \ldots$, $L_{m+1}^{(m)}(0)=p_{m+1}\left(\gamma_{m+1}\right)$ and $L_{m+1}^{(m+1)}(0)=p_{m+2}\left(\gamma_{m+1}\right)$, respectively instead of the constants $a_{m 0}, a_{m 1}, \ldots, a_{m m}$ and $a_{m(m+1)}$ in $x_{m(m+2)}$ which is the bound of $f_{m}^{(m+2)}$ in Theorem $m$. Thus, $h_{m+1}$ provides the inequalities

$$
\left|h_{m+1}^{\prime \prime}(t)\right| \leq y_{(m+1) 2},\left|h_{m+1}^{\prime \prime \prime}(t)\right| \leq y_{(m+1) 3}, \ldots,\left|h_{m+1}^{(m+2)}(t)\right| \leq y_{(m+1)(m+2)}
$$

for all $t$ from Theorem $m$. By Theorem $B$, the solution $f_{m+1}$ of the equivalent equation (2.38) can be written by means of the solution $h_{m+1}$ of (2.39) of the form $f_{m+1}(t)=h_{m+1}(t)-\gamma h_{m+1} * e^{-\gamma t}$. Thus,

$$
\begin{aligned}
& f_{m+1}^{\prime}(t)=h_{m+1}^{\prime}(t)-\gamma e^{-\gamma t}-\gamma h_{m+1}^{\prime} * e^{-\gamma t} \\
& f_{m+1}^{\prime \prime}(t)=h_{m+1}^{\prime \prime}(t)+\gamma^{2} e^{-\gamma t}-\gamma h_{m+1}^{\prime}(0) e^{-\gamma t}-\gamma h_{m+1}^{\prime \prime} * e^{-\gamma t} \\
& f_{m+1}^{\prime \prime \prime}(t)=h_{m+1}^{\prime \prime \prime}(t)-\gamma^{3} e^{-\gamma t}+\gamma^{2} h_{m+1}^{\prime}(0) e^{-\gamma t}-\gamma h_{m+1}^{\prime \prime}(0) e^{-\gamma t}-\gamma h_{m+1}^{\prime \prime \prime} * e^{-\gamma t}
\end{aligned}
$$

and continuing this process, we get the equality

$$
\begin{aligned}
f_{m+1}^{(m+2)}(t)= & h_{m+1}^{(m+2)}(t)+(-1)^{m+2} \gamma^{m+2} e^{-\gamma t}+(-1)^{m+1} \gamma^{m+1} h_{m+1}^{\prime}(0) e^{-\gamma t}+ \\
& +(-1)^{m} \gamma^{m} h_{m+1}^{\prime \prime}(0) e^{-\gamma t}+\cdots-\gamma h_{m+1}^{(m+1)}(0) e^{-\gamma t}-\gamma h_{m+1}^{(m+2)} * e^{-\gamma t} .
\end{aligned}
$$


Thus by taking $\gamma=\gamma_{m+1}=a_{(m+1) 0} /(m+3)$, we have the inequalities

$$
\begin{aligned}
\left|f_{m+1}^{\prime}(t)\right| \leq & \left|h_{m+1}^{\prime}(t)\right|+\gamma+\gamma \int_{0}^{t}\left|h_{m+1}^{\prime}(t-\tau)\right| e^{-\gamma \tau} d \tau \\
\leq & y_{(m+1) 1}+\gamma+\gamma y_{(m+1) 1} \int_{0}^{t} e^{-\gamma \tau} d \tau=2 y_{(m+1) 1}+\gamma-y_{(m+1) 1} e^{-\gamma t} \\
\leq & 2 y_{(m+1) 1}+\gamma=z_{m+1} a_{(m+1) 0}=x_{(m+1) 1}, \\
\left|f_{m+1}^{\prime \prime}(t)\right| \leq & \left|h_{m+1}^{\prime \prime}(t)\right|+\gamma^{2}+\gamma\left|h_{m+1}^{\prime}(0)\right|+\gamma \int_{0}^{t}\left|h_{m+1}^{\prime \prime}(t-\tau)\right| e^{-\gamma \tau} d \tau \\
\leq & y_{(m+1) 2}+\gamma^{2}+\gamma y_{(m+1) 1}+\gamma y_{(m+1) 2} \int_{0}^{t} e^{-\gamma \tau} d \tau \\
= & 2 y_{(m+1) 2}+\gamma^{2}+\gamma y_{(m+1) 1}-y_{(m+1) 2} e^{-\gamma t} \\
\leq & 2 y_{(m+1) 2}+\gamma^{2}+\gamma y_{(m+1) 1}=x_{(m+1) 2} \\
\leq & \left|h_{m+1}^{\prime \prime \prime}(t)\right|+\gamma^{3}+\gamma^{2}\left|h_{m+1}^{\prime}(0)\right|+\gamma\left|h_{m+1}^{\prime \prime}(0)\right|+ \\
& +\gamma \int_{0}^{t}\left|h_{m+1}^{\prime \prime \prime}(t-\tau)\right| e^{-\gamma \tau} d \tau \\
\left|f_{m+1}^{\prime \prime \prime}(t)\right| & y_{(m+1) 3}+\gamma^{3}+\gamma^{2} y_{(m+1) 1}+\gamma y_{(m+1) 2}+\gamma y_{(m+1) 3} \int_{0}^{t} e^{-\gamma \tau} d \tau \\
= & 2 y_{(m+1) 3}+\gamma^{3}+\gamma^{2} y_{(m+1) 1}+\gamma y_{(m+1) 2}-y_{(m+1) 3} e^{-\gamma t} \\
\leq & 2 y_{(m+1) 3}+\gamma^{3}+\gamma^{2} y_{(m+1) 1}+\gamma y_{(m+1) 2}=x_{(m+1) 3} \\
&
\end{aligned}
$$

for all $t$ and by continuing this process, we have

$$
\begin{aligned}
\left|f_{m+1}^{(m+2)}(t)\right| \leq & \left|h_{m+1}^{(m+2)}(t)\right|+\gamma^{m+2}+\gamma^{m+1}\left|h_{m+1}^{\prime}(0)\right|+\gamma^{m}\left|h_{m+1}^{\prime \prime}(0)\right|+ \\
& +\cdots+\gamma\left|h_{m+1}^{(m+1)}(0)\right|+\gamma \int_{0}^{t}\left|h_{m+1}^{(m+2)}(t-\tau)\right| e^{-\gamma \tau} d \tau \\
\leq & y_{(m+1)(m+2)}+\gamma^{m+2}+\gamma^{m+1} y_{(m+1) 1}+\gamma^{m} y_{(m+1) 2}+ \\
& +\cdots+\gamma y_{(m+1)(m+1)}+\gamma y_{(m+1)(m+2)} \int_{0}^{t} e^{-\gamma \tau} d \tau \\
= & 2 y_{(m+1)(m+2)}+\gamma^{m+2}+\gamma^{m+1} y_{(m+1) 1}+\gamma^{m} y_{(m+1) 2}+ \\
& +\cdots+\gamma y_{(m+1)(m+1)}-y_{(m+1)(m+2)} e^{-\gamma t} \\
\leq & 2 y_{(m+1)(m+2)}+\gamma^{m+2}+\gamma^{m+1} y_{(m+1) 1}+\gamma^{m} y_{(m+1) 2}+ \\
& +\cdots+\gamma y_{(m+1)(m+1)} \\
= & b_{(m+1)(m+2)}
\end{aligned}
$$


for all $t$. On the other hand, differentiation of the equivalent equation (2.38) leads to

$$
\begin{aligned}
f_{m+1}^{(m+2)}(t)= & (-1)^{m+2} \gamma^{m+2} e^{-\gamma t}-L_{m+1}(0) f_{m+1}^{(m+1)}(t)-L_{m+1}^{\prime}(0) f_{m+1}^{(m)}(t)- \\
& -L_{m+1}^{\prime \prime}(0) f_{m+1}^{(m-1)}(t)-\cdots-L_{m+1}^{(m)}(0) f_{m+1}^{\prime}(t)- \\
& -L_{m+1}^{(m+1)}(0) f_{m+1}(t)-L_{m+1}^{(m+2)} * f_{m+1}
\end{aligned}
$$

Taking $\gamma=\gamma_{m+1}=a_{(m+1) 0} /(m+3)$, the inequality

$$
\begin{aligned}
\left|f_{m+1}^{(m+2)}(t)\right| \leq & \gamma^{m+2}+L_{m+1}(0)\left|f_{m+1}^{(m+1)}(t)\right|+L_{m+1}^{\prime}(0)\left|f_{m+1}^{(m)}(t)\right|+ \\
& +L_{m+1}^{\prime \prime}(0)\left|f_{m+1}^{(m-1)}(t)\right|+\cdots+L_{m+1}^{(m)}(0)\left|f_{m+1}^{\prime}(t)\right|+ \\
& +L_{m+1}^{(m+1)}(0)\left|f_{m+1}(t)\right|+\int_{0}^{t}\left|L_{m+1}^{(m+2)}(t-\tau)\right|\left|f_{m+1}(\tau)\right| d \tau \\
\leq \quad & \gamma^{m+2}+p_{1}(\gamma) x_{(m+1)(m+1)}+p_{2}(\gamma) x_{(m+1) m}+ \\
& +p_{3}(\gamma) x_{(m+1)(m-1)}+\cdots+p_{m+1}(\gamma) x_{(m+1) 1}+ \\
& +p_{m+2}(\gamma) 2^{m+1}-2^{m+1} \int_{0}^{t} L_{m+1}^{(m+2)}(t-\tau) d \tau \\
= & \gamma^{m+2}+p_{1}(\gamma) x_{(m+1)(m+1)}+p_{2}(\gamma) x_{(m+1) m}+ \\
& +p_{3}(\gamma) x_{(m+1)(m-1)}+\cdots+p_{m+1}(\gamma) x_{(m+1) 1}+ \\
& +2^{m+2} p_{m+2}(\gamma)-2^{m+1} L_{m+1}^{(m+1)}(t) \\
\leq & \gamma^{m+2}+p_{1}(\gamma) x_{(m+1)(m+1)}+p_{2}(\gamma) x_{(m+1) m}+ \\
& +p_{3}(\gamma) x_{(m+1)(m-1)}+\cdots+ \\
& +p_{m+1}(\gamma) x_{(m+1) 1}+2^{m+2} p_{m+2}(\gamma) \\
= & c_{(m+1)(m+2)}
\end{aligned}
$$

is held for all $t$. By $(2.40)$ and $(2.41)$, the bound of $f_{m+1}^{(m+2)}$ is obtained as

$$
\left|f_{m+1}^{(m+2)}(t)\right| \leq \min \left\{c_{(m+1)(m+2)}, b_{(m+1)(m+2)}\right\}=x_{(m+1)(m+2)}
$$

for all $t$. From (2.37),

$$
\begin{aligned}
f_{m+1}^{(m+3)}(t)= & -a_{(m+1) 0} f_{m+1}^{(m+2)}(t)-a_{(m+1) 1} f_{m+1}^{(m+1)}(t)-\cdots- \\
& -a_{(m+1)(m+1)} f_{m+1}^{\prime}(t)-a_{(m+1)(m+2)} f_{m+1}(t)-K_{m+1}^{(m+3)} * f_{m+1} .
\end{aligned}
$$


By the inequalities obtained above and $\left|f_{m+1}(t)\right| \leq 2^{m+1}$, we have

$$
\begin{aligned}
\left|f_{m+1}^{(m+3)}(t)\right| \leq & a_{(m+1) 0} x_{(m+1)(m+2)}+a_{(m+1) 1} x_{(m+1)(m+1)}+\cdots+ \\
& +a_{(m+1)(m+1)} x_{(m+1) 1}+2^{m+1} a_{(m+1)(m+2)}+ \\
& +\int_{0}^{t}\left|K_{m+1}^{(m+3)}(t-\tau)\right|\left|f_{m+1}(\tau)\right| d \tau \\
\leq & a_{(m+1) 0} x_{(m+1)(m+2)}+a_{(m+1) 1} x_{(m+1)(m+1)}+\cdots+ \\
& +a_{(m+1)(m+1)} x_{(m+1) 1}+2^{m+1} a_{(m+1)(m+2)}-2^{m+1} \int_{0}^{t} K_{m+1}^{(m+3)}(\tau) d \tau \\
= & a_{(m+1) 0} x_{(m+1)(m+2)}+a_{(m+1) 1} x_{(m+1)(m+1)}+\cdots+ \\
& +a_{(m+1)(m+1)} x_{(m+1) 1}+2^{m+2} a_{(m+1)(m+2)}-2^{m+1} K_{m+1}^{(m+2)}(t) \\
\leq & a_{(m+1) 0} x_{(m+1)(m+2)}+a_{(m+1) 1} x_{(m+1)(m+1)}+\cdots+ \\
& +a_{(m+1)(m+1)} x_{(m+1) 1}+2^{m+2} a_{(m+1)(m+2)} \\
= & x_{(m+1)(m+3)}
\end{aligned}
$$

for all $t$. Hence, it concludes that if Theorem $n$ is true for $n=m$, it is also true for $n=m+1$. That is to say that Theorem $n$ is valid for all $n \in \mathbb{N}_{2}$.

The infinitely many numbers of kernels $K_{n}$ are of the form

$$
K_{n}(t)=c_{0} t^{n+1}+c_{1} t^{n}+\cdots+c_{n+2} e^{-t}
$$

satisfying all of the conditions of Theorem $n$ were obtained by the method in [8, Example n].

\section{Acknowledgements}

We would like to thank referee for his/her valuable suggestions that have improved the quality of our initial manuscript.

\section{References}

[1] R. S. Anderssen, A. R. Davies, and F. R. de Hoog, Fast collocation methods for Volterra integral equations of convolution type, J. Comput. Appl. Math., 196, (2006), 652-663.

[2] R. S. Anderssen, A. R. Davies, and F. R. de Hoog, The effect of kernel perturbations when solving the interconversion convolution equation of linear viscoelasticity, Appl. Math. Lett., 24, (2011), 71-75. 
Vol. LI (2013) On the Bounds for the Derivatives...

[3] R. Bellman and K. L. Cooke, Differential - Difference Equations, Academic press, New York, 1963.

[4] F. R de Hoog and R. S. Anderssen, Kernel perturbations for a class of secondkind convolution Volterra equations with non-negative kernels, Appl. Math. Lett., 25, (2012), 1222-1225.

[5] R. Ling, Integral equations of Volterra type, J. Math. Anal. Appl., 64, (1978), 381-397.

[6] R. Ling, Solutions of singular integral equations, Internat. J. Math. \&3 Math. Sci., 5 (1), (1982), 123-131.

[7] İ. Özdemir and Ö. F. Temizer, On the linear Volterra integral equations with convolution kernel, XXXVII (2), (1999), 113-122.

[8] í. Özdemir and Ö. F. Temizer, Expansion of the boundaries of the solutions of the linear Volterra integral equations with convolution kernel, Integr. Equ. Oper. Theory, 43(4), (2002), 466-479.

[9] İ. Özdemir and Ö. F. Temizer, The boundaries of the solutions of the linear Volterra integral equations with convolution kernel, Math. Comp., 75, (2006), 11751199.

[10] İ. Özdemir and Ö. F. Temizer, On the solutions of the linear integral equations of Volterra type, Math. Methods Appl. Sci., 30,(18), (2007), 2329-2369.

[11] İ. Özdemir and Ö. F. Temizer, On some properties of the solution of the linear integral equation of Volterra type, An. Univ. Vest Timiş., Ser. Mat.-Inform., (to appear.)

[12] Ö. F. Temizer and İ. Özdemir, On the bounded derivatives of the solutions of the linear Volterra integral equations, Int. J. Comput. Math., 86 (9), (2009), 1512-1541.

[13] F. G. Tricomi, Integral Equations, Dover Publications, Inc., New York, 1985.

Ismet Özdemir

İnönü Üniversitesi Eğitim Fakültesi

44280-Malatya Turkey

E-mail: ismet.ozdemir@inonu.edu.tr

Ö. Faruk Temizer

E-mail: omer.temizer@inonu.edu.tr

Received: 12.10.2012

Accepted: 10.01.2013 